\title{
Linguistic-Valued Approximate Reasoning With Lattice Ordered Linguistic-Valued Credibility
}

\author{
Li Zou and Yunxia Zhang \\ School of Computer and Information Technology, Liaoning Normal University, \\ Dalian 116081, China \\ Xin Liu * \\ School of Mathematics, Liaoning Normal University, \\ Dalian 116029, China \\ Email: liuxin67812@163.com
}

Received 11 January 2014

Accepted 9 June 2014

\begin{abstract}
Linguistic terms are often used to represent the truth degree or credibility degree to manage the uncertainty or imprecision as one of popular ways of knowledge representation in the perception-based decision making problem. This present work represents the credibility of uncertain knowledge using linguistic values. The linguistic-valued credibility is then modeled based on a lattice ordered logical algebra - lattice implication algebra (LIA) and then used in the linguistic-valued based on approximated reasoning. This approach can better express both comparable and incomparable linguistic valued credibility information in knowledge base representation. And it can also fit to approximate reasoning under a fuzzy environment with both comparable and incomparable linguistic credibility. Examples illustrate that the proposed approach can retain the original information and can simulate people's way of thinking through reasoning with words.
\end{abstract}

Keywords: Approximate Reasoning; Knowledge Representation; Linguistic-Valued Credibility Factor; Lattice Implication Algebra.

\section{Introduction}

In the real world, there exists a lot of inaccurate and imperfect information, handling these types of information is an essential feature of intelligence information processing. People often need to make reasoning, judgment and decision in an uncertain environment. In most of cases, people use linguistic terms in natural language to express their opinions and make reasoning and judgments. ${ }^{1-3}$ Many approaches have been presented for representation and reasoning of uncertain knowledge in the last decade. ${ }^{4-7}$ Among various computational approaches in the literature, numerical quantification of linguistic values into a fuzzy set using fuzzy membership functions has played the key role. There exist recently alternative methods to model and compute with linguistic information in natural languages from the different points of view, i.e., fuzzy linguistic approaches or called symbolic approaches, for example, the 2-tuple fuzzy linguistic approaches widely investigated to deal with linguistic-valued decision making problem..$^{8-10,15-16}$ The application of this kind of approaches is beneficial because it introduces a more flexible framework for representing the information in a more direct and suitable way when it is not possible to express it accurately.

Logic is the foundation and standard for justifying or evaluating the soundness and consistency of the methods,

*corresponding author. 
including decision making methods. In order to establish the rational reasoning and decision making approaches to deal with both totally ordered information and non-totally ordered information, it is important and necessary to study the algebraic and logical foundation with such kind of feature for them, which should be some kinds of nonclassical algebraic and logical system.

Lattice implication algebra (LIA) ${ }^{8}$, as a logical algebraic structure, can deal with both comparable and incomparable linguistic values, and also form the basis for lattice-valued logics and logic-based approximate reasoning. ${ }^{11-14}$ Filter and ideal of LIA properties is discussed and basic unary lattice implication algebra equations on the interval sets have been researched. ${ }^{15}$ Lattice implication logic reasoning has been applied to solve the decision making problems. ${ }^{16-17}$

This paper presents a new kind of linguistic-valued approximated reasoning, i.e. representing knowledge credibility directly with linguistic value instead of numerical values during the approximated reasoning process based on knowledge-bases (facts or rules). Linguistic-valued credibility set is modeled based on LIA. The linguistic-valued credibility representation framework is presented and followed by the approximated reasoning scheme which incorporates the linguistic-valued credibility into the reasoning process.

The paper is organized as follows: In Section 2, we briefly review the typical knowledge representation scheme and discuss the uncertain knowledge representation based on linguistic-valued credibility constructed based on LIA. In Section 3, we present a new method of knowledge representation and knowledge reasoning based on LIA-based linguistic-valued credibility set. An example is provided in Section 4 to illustrate the proposed approach. Some conclusions are summarized in Section 5.

\section{Knowledge Representation With Linguistic- Valued Credibility}

Typical knowledge representation method is production rules representation, also called IF-THEN rules. Knowledge-based system contains rules and facts about the knowledge domain covered. Facts or rules in the knowledge-based system can be certain or uncertain based on the information available.

Normally representation of certain knowledge (fact) can be represented as a triple (objects, attributes, values). Representation of uncertain knowledge can be represented as is a 4-tuple (objects, attributes, values, credibility factors). For example, the sentence "John has successful academic achievements." can be expressed as (John, academic achievements, successful). The sentence "John has very successful academic achievements." can be expressed as (John, academic achievements, successful, 0.8), where 0.8 is a credibility factor, which reflects the credibility degree to which John's academic achievements is high. It means the information "John has successful academic achievements." is highly credible. When credibility factor is replaced by 0.4 , it means the credibility degree of to which John's academic achievements is low, even it is not true.

As for the production rule, the basic form is:

$$
\text { IF } P \text { THEN } Q \text {, }
$$

where $P$ is a condition, $Q$ is a conclusion. It means that if the condition is satisfied, then the conclusion $Q$ can be referred. The rule can be certain, for example, considering the rule "IF power on THEN the light will be on", it is certain knowledge (rule in this case). While the following example of a rule

IF windy THEN rain, (0.7)

is a kind of uncertain knowledge (rule). 0.7 means that this knowledge (rule) is not absolutely credible, i.e., there will probably rain if it is windy.

As illustrated above, credibility factors can be used in knowledge (facts or rules) representation so as to better express the meaning of uncertain knowledge, enhance the expressive power of knowledge-base and its applicability, and form the basis to perform uncertainty reasoning. In this paper, we propose to use the linguistic values to represent the credibility degree instead of numerical values, which is more natural in perception-based decision making problem. Considering the different credibility levels in linguistic-valued evaluation and necessary manipulation between different linguistic levels, it is rational to model the linguistic-valued credibility level in terms of logical algebra. So a new description of linguistic valued credibility algebra is introduced below.

Definition 1. Knowledge credibility can be divided into ten levels, i.e., credibility factors set $C=\{$ absolutely credible, very credible, exactly credible, somewhat credible, slight credible, slight incredible, somewhat incredible, exactly incredible, very incredible and absolutely incredible\}. Knowledge representation model about ten linguistic-valued credibility factors is the following 4-tuple: 
(objects, attributes, values, set of ten linguistic-valued credibility factors).

For example, the sentence "John has very successful academic achievements." can be expressed as

(John, academic achievements, successful, very credible).

Here, "very credible" is a linguistic-valued credibility factor.

To better express knowledge, in the model for knowledge representation, the former three in the 4-tuple can be represented descriptive sentences, i.e.,

(John has successful academic achievements, very credible).

Definition 2. If-Then Rule is represented as

IF $\left(P\right.$, Credibility $\left._{1}\right)$ THEN $\left(Q\right.$, Credibility $\left._{2}\right),\left(\right.$ Credibility $\left._{3}\right)$. Here, Credibility ${ }_{1}$, Credibility 2 and Credibility ${ }_{3}$ are the credibility of Evidence $P$, Conclusion $Q$ and this rule respectively. The credibility can be applied into knowledge in accordance with the conditions.

For example,

- IF strongly windy THEN rain, (very credible);

- IF (strongly windy, very credible) THEN (rain, very credible);

- IF (strongly windy, very credible) THEN (rain, somewhat credible), (very credible).

It is a more natural way to deal with and express the credibility of the original knowledge in the form of linguistic values instead of numerical values. Note that some linguistic values are incomparable. How to manage the incomparable linguistic values in the reasoning process is also one problem to be solved in this presented work by using the logical algebra - LIA to model the set of linguistic-valued credibility. Appropriate reasoning result can be obtained from the operations defined in the linguistic truth-valued lattice implication algebra.

There are usually two ways to incorporate the credibility in the description of a sentence. Firstly, linguistic values credibility is added and described alone, for example, Mr. Li is 40 years old. This is very credible. Secondly, linguistic values are described among factual description. For example, "It is very credible that John is 40 years old" can be described as (John, age, 40, very credible). For the above two descriptions, the results are the same.

It is noted that descriptions are not the same when perspectives are different. Suppose "high, strong, and good etc." are positive values. Then the opposites "low, weak, and bad etc." are negative values. In reality many descriptions could be described in a positive way or negative way. For example, "John has successful academic achievements", it describes in a positive way. "John has unsuccessful academic achievements", it describes in a negative way. Comparatively, it is normally easier to deal with it in a positive form in real life and it can also be easier to fit to knowledge reasoning process. So it is necessary to deal with the original knowledge for the sake of knowledge reasoning when the knowledge is described in a negative form. For example, "John has unsuccessful academic achievements". Without dealing with the original knowledge, the sentence can be expressed as:

(John has unsuccessful academic achievements, very credible).

To have better knowledge representation and knowledge reasoning, now consider the original knowledge and make the attribute and the linguistic values factors opposite. For the sake of knowledge reasoning when the knowledge is described in a negative form, the knowledge is represented as follows:

(John has successful academic achievements, very incredible).

So clearly the second form can be more easily handled. Knowledge reasoning is easy to perform when the attribute values of knowledge are classified in a positive way.

Definition 3. ${ }^{8}$ Let $(L, \vee, \wedge, O, I)$ be a bounded lattice with universal boundaries $O$ (the least element) and $I$ (the greatest element) respectively, and " ' " be an orderreversing involution. For any $x, y, z \in L$, if mapping $\rightarrow: L \times L \rightarrow L$ satisfies:

$(I 1): x \rightarrow(y \rightarrow z)=y \rightarrow(x \rightarrow z)$;

$(I 2): x \rightarrow x=I$;

$(I 3): x \rightarrow y=y^{\prime} \rightarrow x^{\prime}$;

(I4): $x \rightarrow y=y \rightarrow x=I$ implies $x=y$;

$(I 5):(x \rightarrow y) \rightarrow y=(y \rightarrow x) \rightarrow x$;

$(I 6):(x \vee y) \rightarrow z=(x \rightarrow z) \wedge(y \rightarrow z)$;

$(I 7):(x \wedge y) \rightarrow z=(x \rightarrow z) \vee(y \rightarrow z)$

Then $(L, \vee, \wedge, ', \rightarrow, O, I)$ is a lattice implication algebra (LIA for short).

Based on LIA with ten linguistic-values, we discuss lattice implication algebra with ten linguistic-valued credibility。 


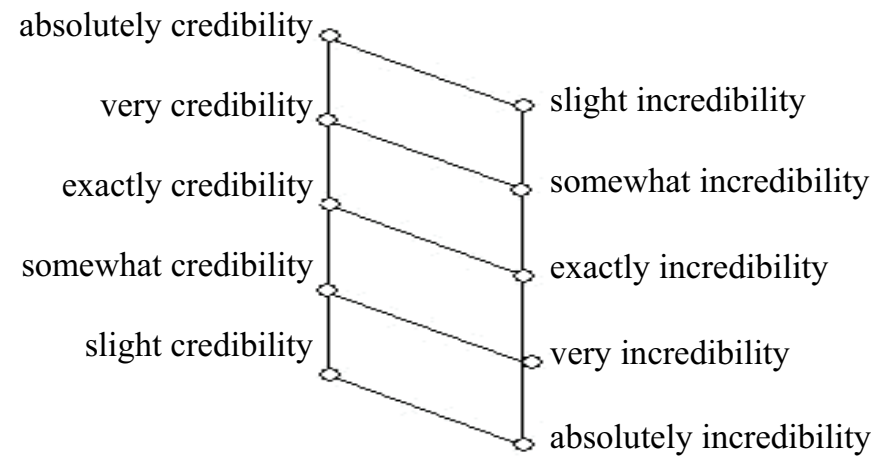

Fig. 1. Hasse diagram of $L_{10}$

Definition 4. In linguistic hedges set $H=\left\{h_{i} \mid i=0,1,2,3,4\right\}$, where $h_{0}$ means "slightly", $h_{1}$ means "somewhat", $h_{2}$ means "exactly", $h_{3}$ means "very" and $h_{4}$ means "absolutely". Evaluating values set is $\left\{L_{1}\right.$, $\left.L_{2}\right\}$, where $L_{1}$ means "credibility" and $L_{2}$ means "incredibility". As for ten-element lattice implication algebra $(V, \vee, \wedge, \rightarrow)$, the operation " $\vee$ "and " $\wedge$ "are shown in Figure 1. And the operation " "' is $\left(h_{\mathrm{i}}, L_{1}\right)^{\prime}=\left(h_{\mathrm{i}}\right.$, $\left.L_{2}\right),\left(h_{\mathrm{i}}, L_{2}\right)^{\prime}=\left(h_{\mathrm{i}}, L_{1}\right)$.

The operation " $\rightarrow$ " is

$$
\left\{\begin{aligned}
\left(h_{\mathrm{i}}, L_{1}\right) \rightarrow\left(h_{\mathrm{j}}, L_{2}\right)=\left(h_{\max \{0, \mathrm{i}+\mathrm{j}-4\}}, L_{2}\right) \\
\left(h_{\mathrm{i}}, L_{2}\right) \rightarrow\left(h_{\mathrm{j}}, L_{1}\right)=\left(h_{\min \{4, \mathrm{i}+\mathrm{j}\}}, L_{1}\right) \\
\left(h_{\mathrm{i}}, L_{1}\right) \rightarrow\left(h_{\mathrm{j}}, L_{1}\right)=\left(h_{\min \{4,4-\mathrm{i}+\mathrm{j}\}}, L_{1}\right) \\
\left(h_{\mathrm{i}}, L_{2}\right) \rightarrow\left(h_{\mathrm{j}}, L_{2}\right)=\left(h_{\min \{4,4-\mathrm{j}+\mathrm{i}\}}, L_{1}\right),
\end{aligned}\right.
$$

Then $(V, \vee, \wedge, \rightarrow)$ is ten linguistic-valued credibility factors LIA.

We obtain the results of operation " $\rightarrow$ " and " " " of ten linguistic-valued credibility factors LIA as table 1 and table 2 .

Table 1. Inverse operation of ten linguistic-valued credibility factors LIA

\begin{tabular}{llllllllllllll}
\hline & $\left(h_{4}, L_{1}\right)$ & $\left(h_{3}, L_{1}\right)$ & $\left(h_{2}, L_{1}\right)$ & $\left(h_{1}, L_{1}\right)$ & $\left(h_{0}, L_{1}\right)$ & $\left(h_{0}, L_{2}\right)$ & $\left(h_{1}, L_{2}\right)$ & $\left(h_{2}, L_{2}\right)$ & $\left(h_{3}, L_{2}\right)$ & $\left(h_{4}, L_{2}\right)$ \\
\hline$\prime$ & $\left(h_{4}, L_{2}\right)$ & $\left(h_{3}, L_{2}\right)$ & $\left(h_{2}, L_{2}\right)$ & $\left(h_{1}, L_{2}\right)$ & $\left(h_{0}, L_{2}\right)$ & $\left(h_{0}, L_{1}\right)$ & $\left(h_{1}, L_{1}\right)$ & $\left(h_{2}, L_{1}\right)$ & $\left(h_{3}\right.$, & $\left.L_{1}\right)$ & $\left(h_{4}\right.$, & $\left.L_{1}\right)$ \\
\hline
\end{tabular}

Table 2. Implication operation of ten linguistic-valued credibility factors LIA

\begin{tabular}{|c|c|c|c|c|c|c|c|c|c|c|}
\hline$\rightarrow$ & $\left(h_{4}, L_{1}\right)$ & $\left(h_{3}, L_{1}\right)$ & $\left(h_{2}, L_{1}\right)$ & $\left(h_{1}, L_{1}\right)$ & $\left(h_{0}, L_{1}\right)$ & $\left(h_{0}, L_{2}\right)$ & $\left(h_{1}, L_{2}\right)$ & $\left(h_{2}, L_{2}\right)$ & $\left(h_{3}, L_{2}\right)$ & $\left(h_{4}, L_{2}\right)$ \\
\hline$\left(h_{4}, L_{1}\right)$ & $\left(h_{4}, L_{1}\right)$ & $\left(h_{3}, L_{1}\right)$ & $\left(h_{2}, L_{1}\right)$ & $\left(h_{1}, L_{1}\right)$ & $\left(h_{0}, L_{1}\right)$ & $\left(h_{0}, L_{2}\right)$ & $\left(h_{1}, L_{2}\right)$ & $\left(h_{2}, L_{2}\right)$ & $\left(h_{3}, L_{2}\right)$ & $\left(h_{4}, L_{2}\right)$ \\
\hline$\left(h_{3}, L_{1}\right)$ & $\left(h_{4}, L_{1}\right)$ & $\left(h_{4}, L_{1}\right)$ & $\left(h_{3}, L_{1}\right)$ & $\left(h_{2}, L_{1}\right)$ & $\left(h_{1}, L_{1}\right)$ & $\left(h_{0}, L_{2}\right)$ & $\left(h_{0}, L_{2}\right)$ & $\left(h_{1}, L_{2}\right)$ & $\left(h_{2}, L_{2}\right)$ & $\left(h_{3}, L_{2}\right)$ \\
\hline$\left(h_{2}, L_{1}\right)$ & $\left(h_{4}, L_{1}\right)$ & $\left(h_{4}, L_{1}\right)$ & $\left(h_{4}, L_{1}\right)$ & $\left(h_{3}, L_{1}\right)$ & $\left(h_{2}, L_{1}\right)$ & $\left(h_{0}, L_{2}\right)$ & $\left(h_{0}, L_{2}\right)$ & $\left(h_{0}, L_{2}\right)$ & $\left(h_{1}, L_{2}\right)$ & $\left(h_{2}, L_{2}\right)$ \\
\hline$\left(h_{1}, L_{1}\right)$ & $\left(h_{4}, L_{1}\right)$ & $\left(h_{4}, L_{1}\right)$ & $\left(h_{4}, L_{1}\right)$ & $\left(h_{4}, L_{1}\right)$ & $\left(h_{3}, L_{1}\right)$ & $\left(h_{0}, L_{2}\right)$ & $\left(h_{0}, L_{2}\right)$ & $\left(h_{0}, L_{2}\right)$ & $\left(h_{0}, L_{2}\right)$ & $\left(h_{1}, L_{2}\right)$ \\
\hline$\left(h_{0}, L_{1}\right)$ & $\left(h_{4}, L_{1}\right)$ & $\left(h_{4}, L_{1}\right)$ & $\left(h_{4}, L_{1}\right)$ & $\left(h_{4}, L_{1}\right)$ & $\left(h_{4}, L_{1}\right)$ & $\left(h_{0}, L_{2}\right)$ & $\left(h_{0}, L_{2}\right)$ & $\left(h_{0}, L_{2}\right)$ & $\left(h_{0}, L_{2}\right)$ & $\left(h_{0}, L_{2}\right)$ \\
\hline$\left(h_{0}, L_{2}\right)$ & $\left(h_{4}, L_{1}\right)$ & $\left(h_{3}, L_{1}\right)$ & $\left(h_{2}, L_{1}\right)$ & $\left(h_{1}, L_{1}\right)$ & $\left(h_{0}, L_{1}\right)$ & $\left(h_{4}, L_{1}\right)$ & $\left(h_{3}, L_{1}\right)$ & $\left(h_{2}, L_{1}\right)$ & $\left(h_{1}, L_{1}\right)$ & $\left(h_{0}, L_{1}\right)$ \\
\hline$\left(h_{1}, L_{2}\right)$ & $\left(h_{4}, L_{1}\right)$ & $\left(h_{4}, L_{1}\right)$ & $\left(h_{3}, L_{1}\right)$ & $\left(h_{2}, L_{1}\right)$ & $\left(h_{1}, L_{1}\right)$ & $\left(h_{4}, L_{1}\right)$ & $\left(h_{4}, L_{1}\right)$ & $\left(h_{3}, L_{1}\right)$ & $\left(h_{2}, L_{1}\right)$ & $\left(h_{1}, L_{1}\right)$ \\
\hline$\left(h_{2}, L_{2}\right)$ & $\left(h_{4}, L_{1}\right)$ & $\left(h_{4}, L_{1}\right)$ & $\left(h_{4}, L_{1}\right)$ & $\left(h_{3}, L_{1}\right)$ & $\left(h_{2}, L_{1}\right)$ & $\left(h_{4}, L_{1}\right)$ & $\left(h_{4}, L_{1}\right)$ & $\left(h_{4}, L_{1}\right)$ & $\left(h_{3}, L_{1}\right)$ & $\left(h_{2}, L_{1}\right)$ \\
\hline$\left(h_{3}, L_{2}\right)$ & $\left(h_{4}, L_{1}\right)$ & $\left(h_{4}, L_{1}\right)$ & $\left(h_{4}, L_{1}\right)$ & $\left(h_{4}, L_{1}\right)$ & $\left(h_{3}, L_{1}\right)$ & $\left(h_{4}, L_{1}\right)$ & $\left(h_{4}, L_{1}\right)$ & $\left(h_{4}, L_{1}\right)$ & $\left(h_{4}, L_{1}\right)$ & $\left(h_{3}, L_{1}\right)$ \\
\hline$\left(h_{4}, L_{2}\right)$ & $\left(h_{4}, L_{1}\right)$ & $\left(h_{4}, L_{1}\right)$ & $\left(h_{4}, L_{1}\right)$ & $\left(h_{4}, L_{1}\right)$ & $\left(h_{4}, L_{1}\right)$ & $\left(h_{4}, L_{1}\right)$ & $\left(h_{4}, L_{1}\right)$ & $\left(h_{4}, L_{1}\right)$ & $\left(h_{4}, L_{1}\right)$ & $\left(h_{4}, L_{1}\right)$ \\
\hline
\end{tabular}

Here are two properties with ten linguistic-valued credibility factors LIA.

- For any $\left(h_{i}, L_{m}\right),\left(h_{j}, L_{n}\right),\left(h_{i}, L_{m}\right) \leq\left(h_{j}, L_{n}\right)$ if and only if $\left(h_{i}, L_{m}\right) \rightarrow\left(h_{j}, L_{n}\right)=\left(h_{4}, L_{1}\right)$;

- $\quad$ For any $\left(h_{i}, L_{m}\right),\left(h_{j}, L_{n}\right),\left(\left(h_{i}, L_{m}\right) \rightarrow\left(h_{j}, L_{n}\right)\right) \vee\left(\left(h_{j}\right.\right.$, $\left.\left.L_{n}\right) \rightarrow\left(h_{i}, L_{m}\right)\right)=\left(h_{4}, L_{1}\right)$.

It can be proved or got from table 2 easily.

Accordingly, the model for knowledge representation with ten linguistic-valued credibility factors LIA is 4tuple:
(Objects, attributes, attribute values, set of ten linguisticvalued credibility factors LIA),

where the element of ten linguistic-valued credibility factors LIA are "absolutely credible", "very credible", "exactly credible", "somewhat credible", "slightly credible ", "slightly credible", "somewhat incredible", "exactly incredible", "very incredible" and "absolutely incredible" respectively.

Then a kind of model for knowledge representation is shown as follows: 
(Objects, attributes, attribute value, $\left(h_{\mathrm{i}}, L_{\mathrm{j}}\right)$ ).

It is easy and feasible to show in this way and it is helpful for the operation in the knowledge reasoning process.

Example 1 Express the following knowledge with the kind of model for knowledge representation with linguistic-valued credibility factors:

- Jack has very successful academic achievements.

(Jack, academic achievements, successful, very credible)

or (Jack has successful academic achievements, $\left(h_{3}\right.$, $\left.L_{1}\right)$ ).

- There will very probably be rain if it is absolutely strongly windy.

IF (it, strongly windy, absolutely credible) THEN (it, rain, very credible)

or IF (strongly windy, $\left.\left(h_{4}, L_{1}\right)\right)$ THEN (rain, $\left(h_{3}, L_{1}\right)$ ).

- It is exactly credible that value $B$ will be somewhat big if Value A is very big.

IF (Value A is big, very credible) THEN (Value B is big, somewhat credible), (exactly credible)

or IF (Value A is big, $\left(h_{3}, L_{1}\right)$ ) THEN (Value B is big, $\left.\left(h_{1}, L_{1}\right)\right),\left(h_{2}, L_{1}\right)$.

- $\quad$ Lily might have got a cold if Lily has a somewhat high fever and is very snotty.

IF (Lily has a fever, $\left(h_{1}, L_{1}\right)$ ) AND (Lily has a running nose, $\left.\left(h_{3}, L_{1}\right)\right)$ THEN (Lily has got a cold, $\left.\left(h_{1}, L_{2}\right)\right)$.

The four cases above have shown how to manage linguistic-valued credibility factors in the IF-THEN rule representation and the feasibility and effectiveness to represent uncertain knowledge with linguistic-valued credibility factors.

\section{Knowledge Reasoning Based on Linguistic- Valued Credibility Factors LIA}

Considering knowledge reasoning to model human being's way of reasoning, it is natural to reasoning with words, i.e., to describe and reasoning directly with linguistic values. The following is the general rule with linguistic-valued credibility (in short LCF):

IF $P\left(h_{i}, L_{m}\right)\left(A N D / O R Q\left(h_{j}, L_{n}\right)\right)$ THEN $R\left(h_{s}, L_{t}\right),\left(h_{u}, L_{v}\right)$.

The following methods are obtained based on ten linguistic-valued credibility factors LIA:

$P\left(h_{i}, L_{m}\right) A N D Q\left(h_{j}, L_{n}\right)=(P$ AND $Q) \quad\left(h_{p}, L_{q}\right)$, where $\left(h_{p}, L_{q}\right)=\left(h_{\mathrm{I}}, L_{m}\right) \wedge\left(h_{j}, L_{n}\right)$;
$P\left(h_{i}, L_{m}\right)$ OR $Q\left(h_{j}, L_{n}\right)=($ P OR $Q)\left(h_{p}, L_{q}\right)$, where $\left(h_{p}\right.$, $\left.L_{q}\right)=\left(h_{i}, L_{m}\right) \vee\left(h_{j}, L_{n}\right)$;

$P\left(h_{p}, L_{m}\right) \rightarrow R\left(h_{i}, L_{n}\right)=(P \rightarrow R)\left(h_{j}, L_{s}\right)$, where $\left(h_{j}, L_{s}\right)=$ $\left(h_{p}, L_{m}\right) \rightarrow\left(h_{i}, L_{n}\right)$;

$\left(E\left(h_{a}, L_{m}\right)\right),\left(h_{b}, L_{n}\right)=E\left(h_{x}, L_{y}\right)$, where $\left(h_{x}, L_{y}\right)=\left(h_{b}\right.$, $\left.L_{n}\right) ' \rightarrow\left(h_{a}, L_{m}\right)$;

Where $E$ means the factor, and $E\left(h_{a}, L_{m}\right)$ means that the factor $E$ Credibility is $\left(h_{a}, L_{m}\right)$.

$E\left(\left(h_{a}, L_{m}\right),\left(h_{b}, L_{n}\right)\right)=E\left(h_{x}, L_{y}\right)$,

where

$$
\left(h_{x}, L_{y}\right)=\left\{\begin{array}{ccc}
\left(h_{\left[\frac{a+b}{2}\right]}, L_{m}\right), & \text { if } & L_{m}=L_{n} ; \\
\left(h_{\left[\frac{a+(4-b)}{2}\right]}, L_{m}\right), & \text { if } & L_{m} \neq L_{n} ;
\end{array}\right.
$$

We can conduct the uncertain knowledge reasoning under the knowledge representation with the linguisticvalued credibility factors. Suppose that the rules in the knowledge-base are given as follows:

$r_{1}:$ IF $E_{1}$ THEN $H$, (absolutely credible)

$r_{2}$ : IF ( $E_{2}$, very credible) THEN $H$, (absolutely credible)

$r_{3}$ : IF ( $E_{3}$, very credible) $A N D$ ( $\left(E_{4}\right.$, somewhat credible $)$ $O R\left(E_{5}\right.$, somewhat incredible) $)$ then $E_{1}$, (very credible)

Once the input for $E_{1}, E_{2}, E_{3}$ and $E_{4}$ are given, we could infer the linguistic-valued credibility of the conclusion $H$.

Firstly, infer the linguistic-valued credibility of evidence $E_{1}$ in accordance to $r_{3}$. Secondly, infer the linguistic-valued credibility of two supporting evidences in accordance to $r_{1}$ and $r_{2}$ respectively, and then infer the final result by combining these two rules using the aggregation operation. The details are specified as follows:

In $r_{3}$, there is certain aggregation relation in evidences, so do the aggregation first:

$$
\left(h_{3}, L_{1}\right) \wedge\left(\left(h_{1}, L_{1}\right) \vee\left(h_{1}, L_{2}\right)\right) .
$$

We can obtain the linguistic-valued credibility of these evidences according to the linguistic-valued credibility factor LIA as: $\left(h_{3}, L_{1}\right)$,

Suppose the credibility value of $E_{1}$ is $X$, thus we have the following logical implication relation:

$$
\left(h_{3}, L_{1}\right) \rightarrow X=\left(h_{3}, L_{1}\right),
$$

Perform the implication operation based on LIA, we get $\operatorname{LCF}\left(E_{1}\right)=\left(h_{2}, L_{1}\right)$; 
We can have the following logical implication relationship in the similar way,

from $r_{1}:\left(h_{2}, L_{1}\right) \rightarrow Y=\left(h_{4}, L_{1}\right)$;

from $r_{2}:\left(h_{3}, L_{1}\right) \rightarrow Z=\left(h_{4}, L_{1}\right)$;

Then we get $L C F(H)=\left(h_{2}, L_{1}\right)$ or $\left(h_{3}, L_{1}\right)$ or $\left(h_{4}, L_{1}\right)$ according to $r_{1}$ and $L C F(H)=\left(h_{4}, L_{1}\right)$ or $\left(h_{3}, L_{1}\right)$ according to $r_{2}$;

At last, operate the two credibility values in accordance with Eq. (5), and we can obtain the final inferred result: $\left(h_{2}, L_{1}\right)$ or $\left(h_{3}, L_{1}\right)$ or $\left(h_{4}, L_{1}\right)$, i.e., the conclusion $H$ is at least exactly credible and at most absolutely credible.

In Example 1, we have obtained the multiple solutions but not a single value because of the uncertainty of the rules and the factors. The reasoning process is more intuitive and the result is easier to understand with linguistic-valued credibility factors.

In the reality there are many factors bring the uncertainty, such as fuzzy description that will cause uncertain knowledge and results in multiple solutions for the same conclusion attribute.

Example 2. Considering an example to evaluate the risk an individual brings from the traffic accident. Here we know, an exactly middle-aged or very careful person is exactly attentive. The one absolutely not tired and very attentive won't bring the traffic accident. And the one with very good driving skill won't bring the traffic accident with absolutely (Figure 2). Mr. Bob is an exactly middle-aged and very careful man and exactly good at driving. Today he is somewhat tired.

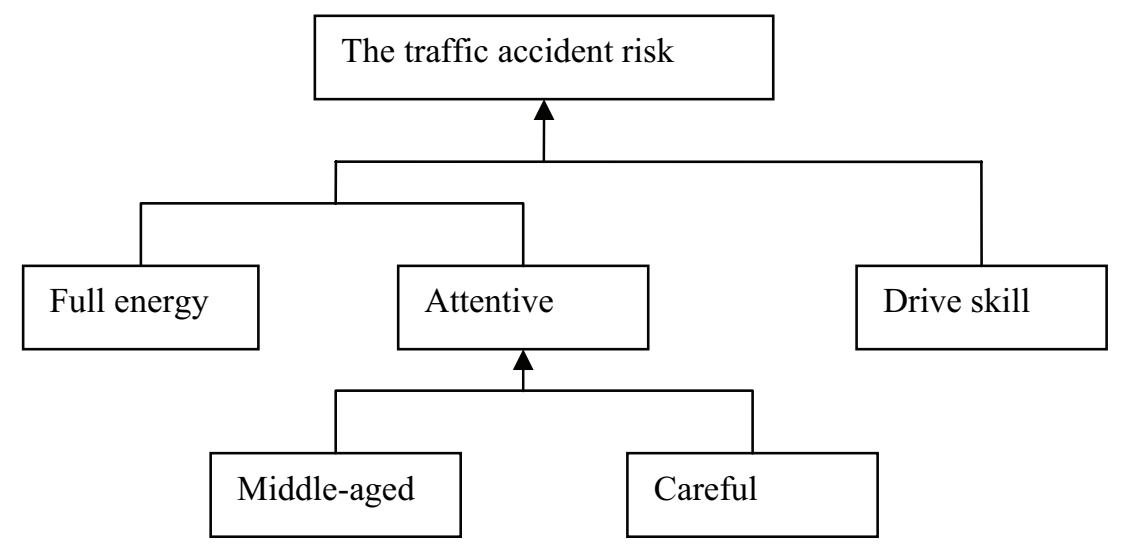

Fig. 2 The traffic accident risk analysis

Then the rules above can be described as follow:

$r_{1}$ : IF not tired and attentive THEN he won't bring traffic accident, (absolutely credible);

$r_{2}$ : IF good drive skill THEN he won't bring traffic accident, (absolutely credible);

$r_{3}$ : IF middle-aged or careful THEN he is attentive, (very credible);

About Mr. Bob we know the knowledge represented with linguistic-valued credibility factors, i.e., (Middleaged, exactly credible), (careful, very credible), (good dive skill, exactly credible), and (not tired, somewhat credible)

The uncertain rules can be represented with linguisticvalued credibility factors as follows: $r_{1}$ : IF (not tired, absolutely credible) and (attentive, very credible) THEN (he won't bring traffic accident, very credible)

$r_{2}$ : IF (good drive skill, very credible) THEN (he won't bring traffic accident, absolutely credible)

$r_{3}$ : IF (middle-aged, exactly credible) or (careful, very credible) THEN (he is attentive, exactly credible)

Then we can update each rule's credibility as follows:

$r_{1}:\left(\left(h_{4}, L_{1}\right) \wedge\left(h_{3}, L_{1}\right)\right) \rightarrow\left(h_{3}, L_{1}\right)=\left(h_{4}, L_{1}\right) ;$

$r_{2}:\left(h_{3}, L_{1}\right) \rightarrow\left(h_{4}, L_{1}\right)=\left(h_{4}, L_{1}\right)$

$r_{3}:\left(\left(h_{2}, L_{1}\right) \vee\left(h_{3}, L_{1}\right)\right) \rightarrow\left(h_{2}, L_{1}\right)=\left(h_{3}, L_{1}\right)$.

We can obtain the credibility of that Bob is attentive from the rule $r_{3}$. 


$$
\left(\left(h_{2}, L_{1}\right) \vee\left(h_{3}, L_{1}\right)\right) \rightarrow X=\left(h_{3}, L_{1}\right) .
$$

We get $L C F($ attentive $)=\left(h_{2}, L_{1}\right)$, that is, (attentive, exactly credible).

From the rule $r_{1}$ and the rule $r_{2}$ we can obtain the credibility that Bob won't bring traffic accident.

From the rule $r_{1}$ :

$$
\left(\left(h_{1}, L_{2}\right) \wedge\left(h_{3}, L_{1}\right)\right) \rightarrow Y=\left(h_{4}, L_{1}\right) .
$$

We get $L C F$ (Bob won't bring traffic accident $)=\left(h_{1}, L_{2}\right)$, $\left(h_{3}, L_{1}\right),\left(h_{0}, L_{2}\right)$ or $\left(h_{4}, L_{1}\right)$. So Bob won't bring traffic accident at least somewhat incredibly, represented as (Bob won't bring traffic accident, somewhat incredible).

From the rule $r_{2}$ :

$$
\left(h_{2}, L_{1}\right) \rightarrow Z=\left(h_{4}, L_{1}\right)
$$

We get $L C F$ (Bob won't bring traffic accident) $=\left(h_{2}, L_{1}\right)$, $\left(h_{3}, L_{1}\right),\left(h_{4}, L_{1}\right)$. So Bob won't bring traffic accident at least exactly credible, represented as (Bob won't bring traffic accident, exactly credible).

By Eq. (5), we can aggregate the results of the two respective rules. The result is $L C F$ (Bob won't bring traffic accident $)=\left(h_{2}, L_{1}\right)$. Bob today won't bring traffic accident exactly credibly if he drives out. So Bob shouldn't drive out because it is exactly dangerous. It is coincident with our common knowledge that if a person is somewhat tired and has exactly good drive skill even if he is very careful, he would bring traffic accident more than exactly.

\section{Case Study}

As far as knowledge representation is concerned, the predicates in the conditions and the conclusions named "be1" and the predicate of the rule named "be 2 " are used to handle uncertain and imprecise knowledge. A fact like " $P\left(h_{i}, L_{m}\right)$ " is represented by the clause:

$$
\text { be } 1\left(P,\left(h \_i, L \_m\right)\right) \text {, }
$$

and a rule like "IF $P\left(h_{i}, L_{m}\right)$ THEN $R\left(h_{u}, L_{v}\right)$." is represented by the clause:

$$
b e 2\left(R,\left(h \_u, L \_y\right)\right):-b e 1\left(P,\left(h \_i, L \_m\right)\right)
$$

and a rule like "IF $P\left(h_{i}, L_{m}\right)$ THEN $R\left(h_{s}, L_{t}\right),\left(h_{u}, L_{v}\right)$." is represented by the clause:

$$
\text { be2(be1 } \left.\left(R,\left(h \_s, L \_t\right)\right),\left(h \_u, L \_y\right)\right):-b e 1\left(P,\left(h \_i, L \_m\right)\right)
$$

For example, the methods obtained based on ten linguistic-valued credibility factors LIA (1)-(5) can be represented as below.

(1) bel $\left(P,\left(h \_i, L \_m\right)\right)$ AND bel $\left(Q,\left(h j j, L \_n\right)\right)=b e 1(O$, $\left.\left(h \_p, L \_q\right)\right)$ where $\left(h \_p, L \_q\right)=\left(h \_i, L \_m\right) \wedge\left(h \_j, L \_n\right)$, and $O=P A N D Q$;

(2) be $1\left(P,\left(h \_i, L \_m\right)\right)$ OR be $1\left(Q,\left(h \_j, L \_n\right)\right)=b e 1(O$, $\left.\left(h \_p, L \_q\right)\right)$ where $\left(h \_p, L \_q\right)=\left(h \_i, L \_m\right) \vee\left(h \_j, L \_n\right)$, and $O=P O R Q$;

(3) $b e 1\left(P,\left(h \_i, L \_m\right)\right) \rightarrow \operatorname{be} 1\left(R,\left(h \_s, L \_t\right)\right)=b e 1\left(O,\left(h \_p\right.\right.$, $\left.\left.L \_q\right)\right)$ where $\left(h \_p, L \_q\right)=\left(h \_i, L \_m\right) \rightarrow\left(h \_s, L \_t\right)$, and $O=P \rightarrow R$;

(4) $b e 2\left(b e 1\left(E,\left(h \_a, L \_b\right)\right),\left(h \_u, L \_v\right)\right)=b e 1\left(E,\left(h \_x\right.\right.$, $\left.\left.L \_y\right)\right)$ where $\left(h \_x, L \_y\right)=\left(h \_a, L \_b\right) \rightarrow\left(h \_u, L \_v\right)$;

(5) $A G G\left(b e 1\left(E,\left(h a, L \_b\right), b e 1\left(E,\left(h \_u, L \_v\right)\right)=b e 1(E\right.\right.$, $\left.\left(h \_x, L_{-} y\right)\right)$, where $\left(h \_x, \bar{L} \_y\right)=\left(h \_(a+u) / 2, L_{-} 1\right)$, where $\mathrm{A} \overline{\mathrm{GG}}$ is a function to aggregate several uncertain facts with multiple credibility values.

Example 3 Considering a social risk assessment system shown in Figure 3.

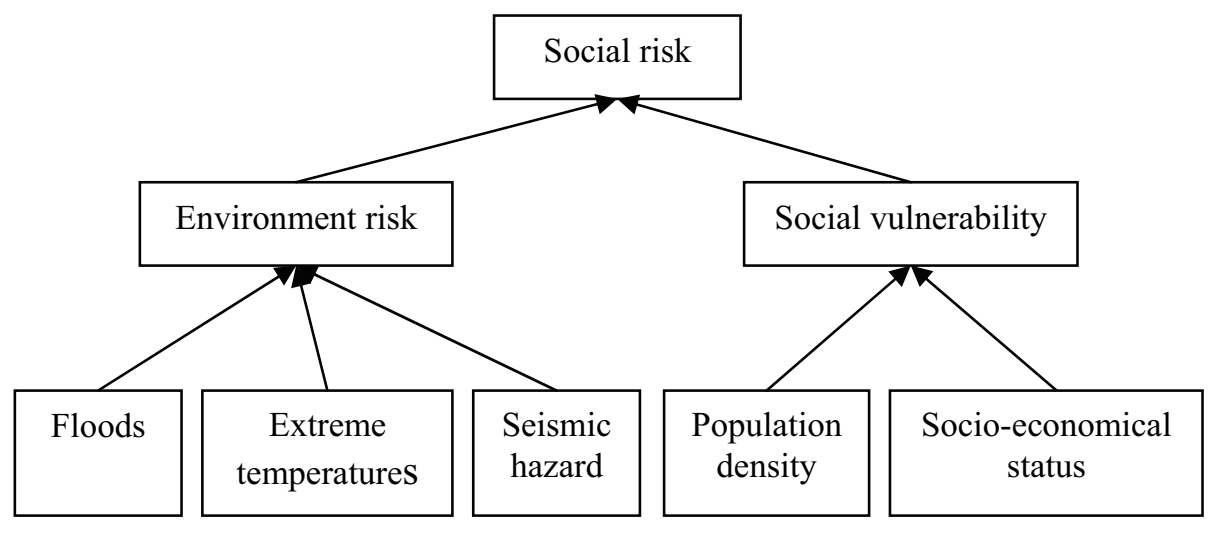


Fig. 3 A social risk assessment system

The social risk assessment system is with five inputs and one output. The output is "Social risk" and the inputs are "Seismic hazard", "Population density", "Socioeconomical status", "floods", and "Extreme temperatures". The credibility rules in the risk assessment are defined as follow:

$r_{1}$ : IF ((Floods, exactly credible) AND (Extreme temperatures, very credible)) $O R$ (Seismic hazard, very credible) THEN Environment risk (absolutely credible); $r_{2}$ : IF (Population density, very credible) AND (Socioeconomical status, somewhat incredible) THEN Social vulnerability (exactly credible);

$r_{3}$ : IF Environment risk THEN Social risk (very credible); $r_{4}$ : IF Social vulnerability THEN Social risk (absolutely credible).

In this case study, the output "Social risk" is named as $H$, and the factors infecting the social risk is named as $E_{-} 1$, $E \_2, E \_3, E \_4, E \_5, E \_6, E \_7$, respectively as shown in Table 3 .

Table 3. Factors and conclusion in the social risk assessment system

\begin{tabular}{|c|c|c|c|}
\hline Social risk & $\begin{array}{c}\text { Environment } \\
\text { risk }\end{array}$ & $\begin{array}{c}\text { Social } \\
\text { vulnerability }\end{array}$ & Floods \\
\hline$H$ & $E \_1$ & $E \_2$ & $E 3$ \\
\hline $\begin{array}{c}\text { Extreme } \\
\text { temperatures }\end{array}$ & $\begin{array}{c}\text { Seismic } \\
\text { hazard }\end{array}$ & $\begin{array}{c}\text { Population } \\
\text { density }\end{array}$ & $\begin{array}{c}\text { Socio- } \\
\text { economical status }\end{array}$ \\
\hline$E 44$ & $E \_5$ & $E \_6$ & $E$ 7 \\
\hline
\end{tabular}

(1) Write the conjecture:

? bel $(H$, value $)$.

(2) Write the rules:

-be2(E_1,(h_4,L_1)) :-be1 $\left(E \_3,\left(h \_2, L \_1\right)\right) A N D$

be1(E_4, (h_3, L_1)) OR be1(E_5, (h_3,L_1)).

-be2(E_2,(h_2,L_1)) :-be1 $\left(E \_6,\left(h \_3, L \_1\right)\right) A N D$

be1 $\left(E \_7,\left(h \_1, L \_2\right)\right)$.

-be2(H,(h_3, L_1)) :-be1(E_1,value_1).

-be2(H,(h_4,L_1)) :-be1(E_2,value_2).

-stop.

(3) Intermediate conclusion:

-be1 $\left(E \_1,\left(h \_3, L \_1\right)\right)$.

(4) Partial final conclusion:

- be1(H, (h_2, L_1)).

(5) Intermediate conclusion:

-be1(E_2, (h_2,L_1)).

(6) Partial final conclusion:

- be1 $\left(H,\left(h \_2, L \_1\right)\right)$.

(7) final conclusion:

-be1 $\left(H,\left(h \_2, L \_1\right)\right)$.
It should be noted that the final conclusion is the least credibility. Therefore, the social risk is more than exactly high.

Example 2 and Example 3 illustrate the effectiveness and feasibility of knowledge representation and knowledge reasoning based on linguistic-valued credibility. Due to the fact that the operations used in the reasoning process are based on the operations defined in a logical algebra -LIA, which are again based on logical axioms and have the strict theoretical logical foundation. This justifies the rationality of the proposed uncertainty knowledge reasoning with linguistic-valued credibility.

\section{Conclusions}

This paper focused on the linguistic-valued representation of credibility appeared in the knowledge-base (facts and rules) and proposed to model these linguistic-valued credibility values using a lattice-ordered logical algebra structure. It was followed by the general knowledge representation and knowledge reasoning framework and approaches with linguistic-valued credibility factors. Examples illustrated that the proposed approach can make it not only easier to understand the knowledge with the model for knowledge representation but also more effective to perform knowledge-based reasoning. Based on linguistic-valued credibility factors lattice implication algebra, the reasoning of different kinds of credibility can be solved by using different operations of linguisticvalued credibility factors. This will place some 
theoretical support for linguistic-valued involved perception-based decision making problem in order to achieve reason with words.

\section{Acknowledgments}

This work is partly supported by National Nature Science Foundation of China (Grant No. 61105059, 61175055, 61372187 and 61173100), Sichuan Key Technology Research and Development Program under Grant No.2011FZ0051 and Sichuan Key Laboratory of Intelligent Network Information Processing (SGXZD1002-10).

\section{References}

1. L. Martinez, D. Ruan F. and Herrera, Computing with words in decision support systems: an overview on models and applications, International Journal of Computational Intelligence Systems. 3(4) (2010) 382-395.

2. F. Herrera and L. Martinez, The 2-tuple fuzzy linguistic computational model: advantages of its linguistic description, accuracy and consistency, International Journal of Uncertainty, Fuzziness and Knowledge-Based Systems. 9(2001) 33-48.

3. F. Herrera, S. Alonso, F. Chiclana and E. Herrera-Viedma, Computing with words in decision making: foundations, trends and prospects, Fuzzy Optimization and Decision Making. 8(4) (2009) 337-364.

4. N.C. Ho, A topological completion of refined hedge algebras and a model of fuzziness of linguistic terms and hedges, Fuzzy Sets and Systems. 158(2007) 436-451.

5. N.C. Ho and N. V. Long, Fuzziness measure on complete hedge algebras and quantifying semantics of terms in linear hedge algebras, Fuzzy Sets and Systems. 158(2007) $452-471$.

6. Z. Pei, D. Ruan, J. Liu and Y. Xu, Linguistic Values based Intelligent Information Processing: Theory, Methods, and Application, Atlantis Computational Intelligence Systems. 2009 (Atlantis press \& World Scientific).
7. Z. Pei, Y. Xu, D. Ruan and K. Qin, Extracting complex linguistic data summaries from personnel database via simple linguistic aggregations, Information Sciences. 179(2009) 2325-2332.

8. Y. Xu, D. Ruan, K.Y. Qin and J. Liu, Lattice-Valued Logic: An Alternative Approach to Treat Fuzziness and Incomparability, (Springer-Verlag, Heidelberg, 2003).

9. Y. Xu, J. Liu, D. Ruan and T.T. Lee, On the consistency of rule bases based on lattice-valued first-order logic $L F(X)$, International Journal of Intelligent Systems. 21(2006) 399424.

10. L. Zou, D. Ruan, Z. Pei and Y. Xu, A linguistic truthvalued reasoning approach in decision making with incomparable information, Journal of Intelligent and Fuzzy Systems, 19(4-5) (2008) 335-343.

11. L. Zou, Z. Pei, X. Liu and Y. Xu, Semantics of linguistic truth-valued intuitionistic fuzzy proposition calculus, International Journal of Innovative Computing, Information and Control. 5(12) (2009) 4745-4752.

12. L. Zou, X. Liu, Z.Wu and Y. Xu, A uniform approach of linguistic truth values in sensor evaluation, International Journal of Fuzzy Optimization and Decision Making. 7(4) (2008) 387-397.

13. L. Zou, D. Ruan, Z. Pei and Y. Xu. A linguistic-valued lattice implication algebra approach for risk analysis, Journal of Multi-Valued Logic and Soft Computing. 17(4) (2011) 293-303.

14. J. Liu, W.J. Li, S.W. Chen and Y. Xu. An axiomatizable logical foundation for lattice-ordered qualitative linguistic approach for reasoning with words, Information Sciences. 262 (2014) 110-125.

15. Du Hao Cui, Sun Bin and Yao Ying Le. Research of unary lattice implication algebra equations on interval sets, Advanced Materials Research, 850-851 (2014) 761-766.

16. S.W. Chen, J. Liu, H. Wang, Y. Xu and J.C. Augusto, A linguistic multi-criteria decision making approach based on logical reasoning, Information Sciences, 258 (2013) 266276.

17. S.W. Chen, J. Liu, H. Wang and J.C. Augusto, Ordering based decision making: a survey, Information Fusion Journal, in press, available online 25 October 2012. 